\title{
SINGLE-SENSOR IMAGE COMPRESSION FROM THE END-USER'S PERSPECTIVE
}

\author{
Rastislav Lukac \\ Multimedia Laboratory, Dept. of ECE \\ University of Toronto, 10 King's College Road \\ Toronto, M5S 3G4, ON Canada \\ e-mail: lukacr@ieee.org
}

\author{
Konstantinos N. Plataniotis \\ Multimedia Laboratory, Dept. of ECE \\ University of Toronto, 10 King's College Road \\ Toronto, M5S 3G4, ON Canada \\ e-mail: kostas@dsp.utoronto.ca
}

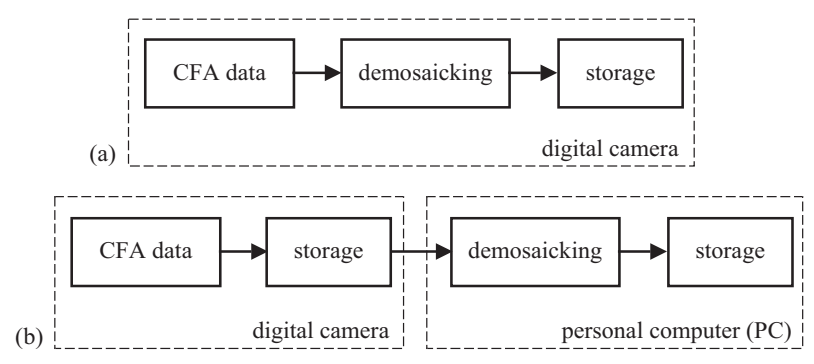

Figure 1. Basic single-sensor imaging solutions. Camera image is stored in: (a) full-color demosaicked format, (b) CFA format.

\section{Introduction}

Most, if not all, consumer digital cameras store captured images using the Exchangeable Image File (EXIF) format [1] or the Tagged Image File Format for Electronic Photography (TIFF-EP) [2]. Both storage formats use Joint Photographic Experts Group (JPEG) standard as a default image compression solution and allow for addition of the metadata information, e.g. about the camera and the environment. However, EXIF-based digital cameras store the image in the demosaicked full-color format, whereas camera working in the TIFF-EP mode store the so-called color filter array (CFA) data.

This paper presents single-sensor image compression solutions suitable for CFA-based digital cameras. Operating either on the acquired gray-scale CFA data or the restored full-color demosaicked data, the proposed solutions offer different design characteristics and performance. Thus, the order of the compression and demosaicking steps in the single-sensor imaging pipeline influences the visual quality of the final images determined for displaying and/or printing. Considered basic configurations are based on: i) demosaicking followed by full-color image compression, ii) straightforward CFA image compression followed by demosaicking, or iii) compression of the preprocessed CFA data followed by demosaicking. To cover a wide range of design and performance issues which are essential in single-sensor imaging, well-known demosaicking solutions and two coding schemes (JPEG, JPEG 2000) will be used at various compression ratios to demonstrate the side effects of the

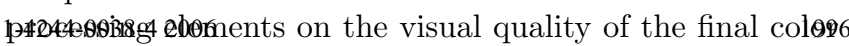
rREEB GFFEgECGEI, Ottawa, May 2006

\section{Single-Sensor Imaging Pipeline}

The camera acquires the visual scene by first focusing and transmitting the light through the CFA and then sampling the visual information using the charge-coupled device $(\mathrm{CCD})$ or complementary metal oxide semiconductor (CMOS) sensor, and an analog-to-digital (A/D) converter [3]. Since the sensor is a monochromatic device, the raw single-sensor data constitutes a gray-scale, mosaic-like, image. The full-color information is restored from the acquired samples using demosaicking [3]-[5].

As it can be seen from the simplified block schemes in Fig.1 [6], demosaicking can be part of the processing pipeline implemented in the camera hardware/software or it can be performed in a companion personal computer (PC) which interfaces with the digital camera. In the former case, the camera stores demosaicked full-color images using EXIF standard. In the latter case, TIFF-EP standard is used to store the raw CFA data. The conventional processing pipeline (Fig.1a) should operate in the real time whereas its PC-based variant (Fig.1b) allows the utilization of sophisticated processing solutions and the end-user control of processing steps to achieve the required visual quality.

In both EXIF and TIFF-EP, the default image filerecording format is strictly based on existing formats used by commercial applications to utilize available functions for viewing and manipulating the images. Overview of EXIF and TIFF-EP standards in [7] reveals that camera manufacturers mainly rely on lossy JPEG-type compression applied either to the full-color demosaicked image or to the gray-scale CFA data. 


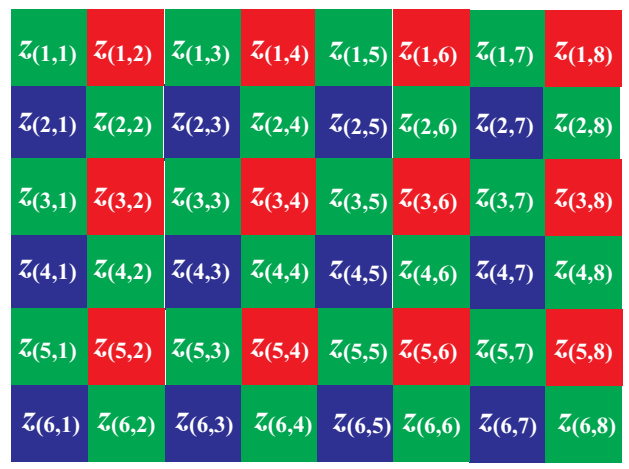

Figure 2. Popular Bayer CFA with the GRGR phase in the first row.

Following this trend, a JPEG coding scheme [8] is employed in the considered single-sensor processing pipelines. Advanced variants of the solutions presented in this paper use JPEG 2000 [9] as it is a new standard for still image compression. Overcoming the difficulties with the computational complexity and memory requirements should allow the inclusion of JPEG 2000 in the next generation of digital cameras $[5],[7],[9]$, as the standard enhances color space flexibility and improved metadata support. Therefore, it is important to evaluate and analyze performance improvements which can be obtained by replacing JPEG with JPEG 2000 in the single-sensor imaging pipeline.

To demonstrate the concept and evaluate the performance, a number of CFAs, with an overview to be found in [3], can be used. In this paper, we use the popular Bayer CFA shown in Fig.2 which is commonly used in the research community for its good relative performance and simplicity of color restoration.

\subsection{Demosaicked Image Compression}

Most consumer digital cameras first demosaick the acquired CFA data and then compress the demosaicked fullcolor image using the conventional color image compression methods to reduce redundancy in color RGB data [1],[7][9]. However, compression of demosaicked images can be counterproductive since demosaicking triples the amount of the data to be compressed by populating the two missing color components at each spatial location of the acquired CFA image. On the other hand, color image compression aims to de-correlate the data. Therefore, this approach may reduce compression ratios and increase the computational complexity.

\subsection{CFA Image Compression}

Compressing directly the CFA data rather than the demosaicked image basically results in a three-fold reduction of the information to be coded [10]-[13]. Moreover, decompressing the CFA image in its original quality or wiolar a small compression error allows to obtain a high-quality
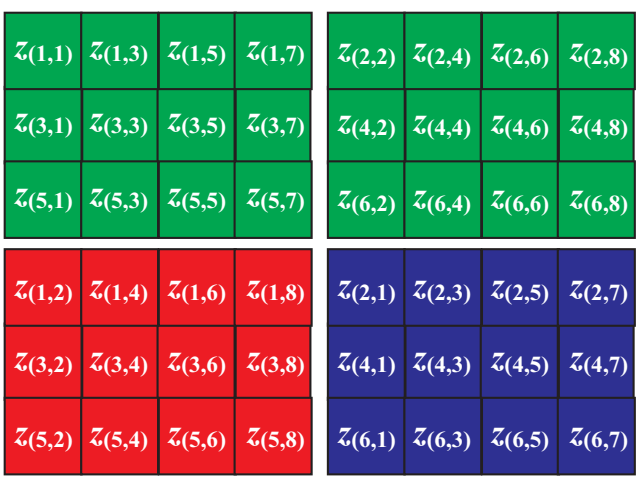

Figure 3. CFA image re-arrangement used to eliminate artificial high-frequency due to the mosaic layout.

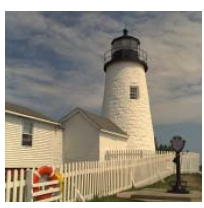

(a)

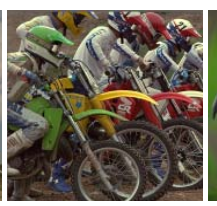

(b)

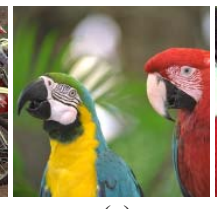

(c)

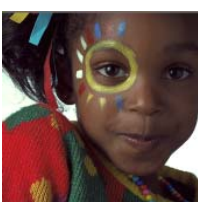

(d)
Figure 4. $512 \times 512$ test color images: (a) Lighthouse, (b) Bikes, (c) Parrots, (d) Face.

demosaicked image by running computationally expensive, sophisticated demosaicking algorithms using a companion PC. Therefore, this approach is used in high-end cameras and applications where the final visual quality is of paramount importance [7].

However, operating on the original mosaic layout may limit the compression efficiency due to the artificial high frequencies between acquired color components in the CFA image. To overcome the problem and achieve higher compression ratios, CFA data should be pre-processed or rearranged prior to compression in order to obtain pixel arrangements more appropriate for image coding [7],[11],[13]. In this paper, we will use the approach depicted in Fig.3. Thus, the original CFA image is re-arranged into four sub-images to be cost-effectively stored and/or potentially transmitted to the end-user. After decoding, the subimages are re-arranged back into the original CFA layout to restore the full-color information and produce the final color RGB image determined for visual inspection.

\section{Experimental Results}

To determine the performance of presented single-sensor compression solutions, a number of test images have been utilized. Examples are shown in Fig.4. The test color images which vary in color appearance and the complexity of the structural content (edges and fine details) have been captured using a three-sensor digital camera and normalized to an 8-bit per channel RGB representation and $512 \times 512$ spatial resolution. 

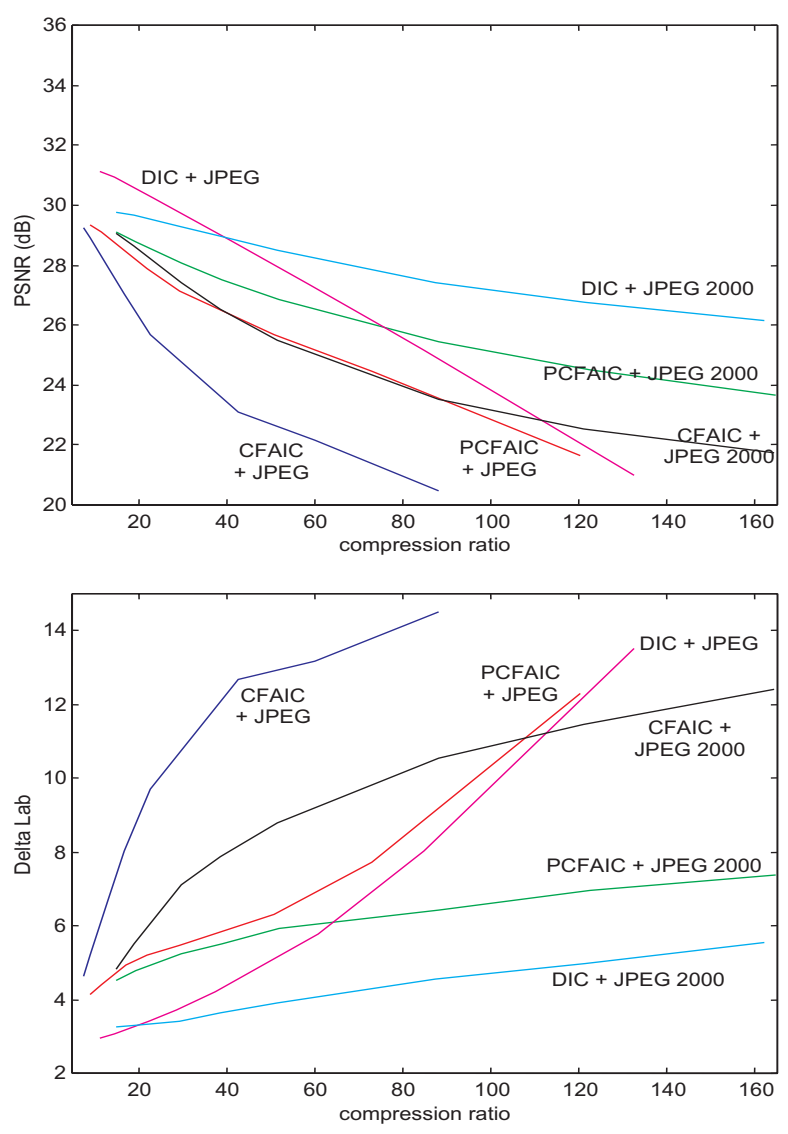

Figure 5. Performance evaluation using the BI demosaicking scheme: (top) PSNR, (bottom) $\Delta_{L a b}$.

The tests were performed by sampling the original images (Fig.4) with a GRGR-phased Bayer CFA filter (Fig.2) to obtain a plain CFA image. In the so-called demosaicked image compression (DIC) pipeline, the CFA image is demosaicked, coded, decoded and displayed. In the CFA image compression (CFAIC) pipeline, the CFA image is coded, decoded, demosaicked and displayed. Finally, in the preprocessing-based CFA image compression (PCFAIC) pipeline, the CFA image is preprocessed, coded, decoded, inversely preprocessed, demosaicked and displayed. Note that both CFAIC and PCFAIC treat respectively the CFA image and its re-arranged variant as a gray-scale image. In all three considered pipelines, the demosaicked full-color images were respectively obtained using the bilinear interpolation (BI) scheme [14] and the color-correlation adaptive (CCA) demosaicking scheme [15]. Performance was measured by comparing the original full color images to the final, displayed images. To facilitate objective comparisons, the peak signal-to-noise ratio (PSNR) criterion defined in the RGB color space and $\Delta_{L a b}$ criterion defined in the perceptually uniform CIE Lab color space are useelas The definition of these criteria can be found in [7].
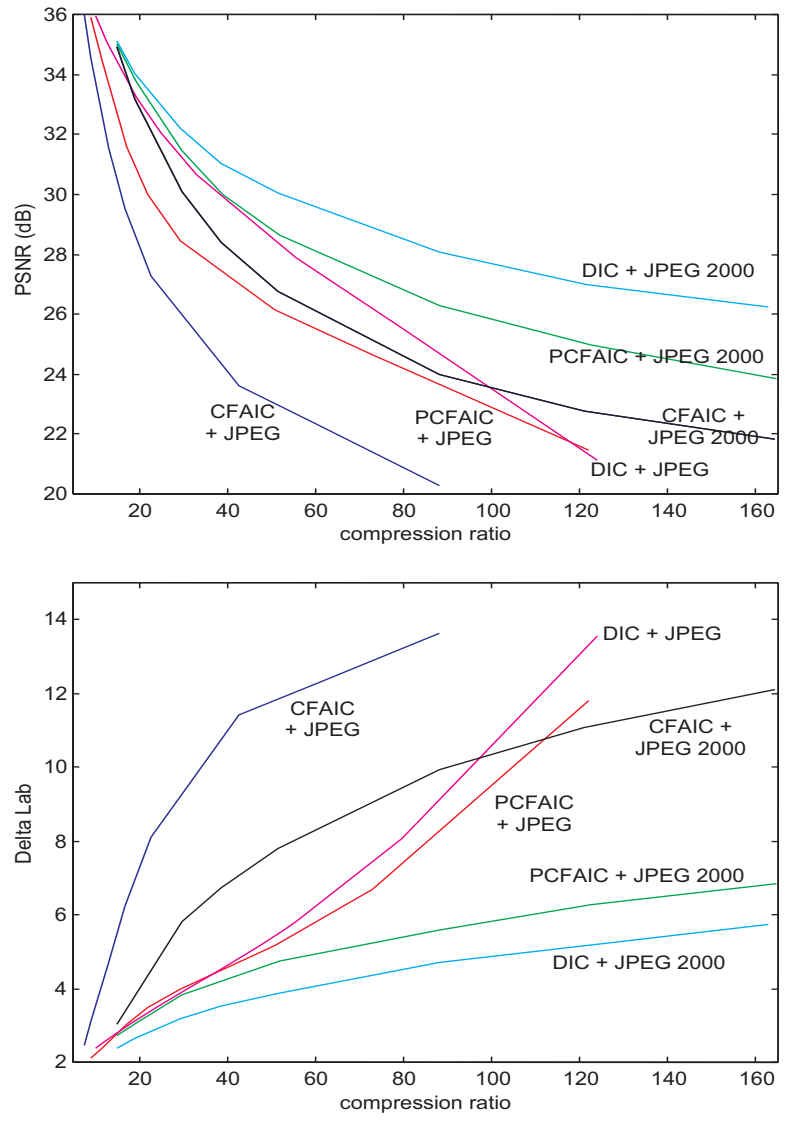

Figure 6. Performance evaluation using the CCA demosaicking scheme: (top) PSNR, (bottom) $\Delta_{L a b}$.

Figs.5-6 show the results calculated, using eight compression ratio values per curve, as aggregated measures averaged over the images in Fig.4. As it can be seen, the choice of the demosaicking solution in any processing pipeline is essential at compression ratios under 80. Increasing the ratio value up this range usually reduces the performance of powerful demosaicking solutions, such as the CCA scheme (Fig.6), down to simple demosaicking solutions, such as the BI scheme (Fig.5). Further inspection of the error curves reveals that all JPEG 2000-based pipelines outperformed their variants equipped with the JPEG coding scheme in terms of both compression ratios and achieved quality. The results also show that among the considered processing solutions the DIC pipeline, i.e. demosaicking followed by fullcolor image compression, is far the best options to reduce processing errors while straightforward CFA image compression in CFAIC scheme results in the worst performance. However, it should be noted that the DIC approach requires to code triple amount of data compared to CFA-based compression. Thus, cost-effective devices should rather use the PCFAIC pipeline which produces similar visual quality (see $\Delta_{L a b}$ values) as the DIC pipeline. 


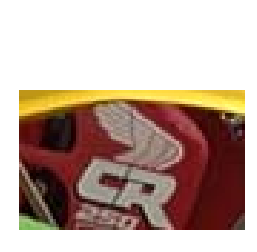

(a)

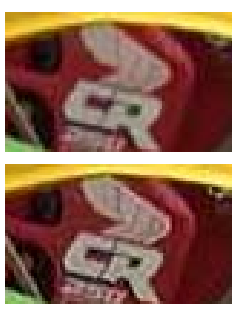

(b)

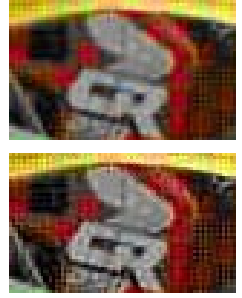

(c)

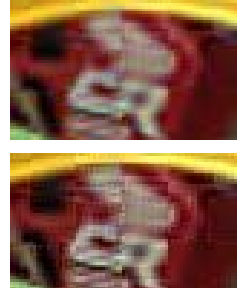

(d)

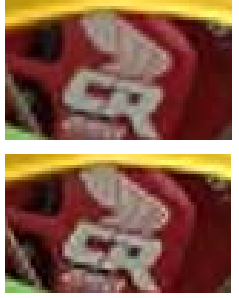

(e)

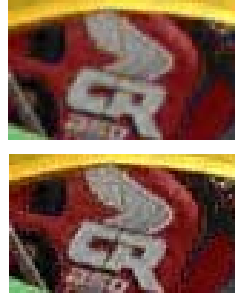

(f)

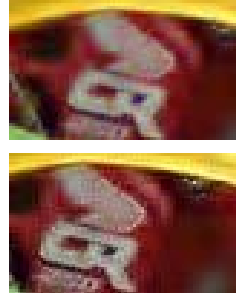

(g)

Figure 7. Achieved results: (top) BI demosaicking scheme, (bottom) CCA demosaicking scheme, (a) original image, (b-d) JPEG, (e-g) JPEG 2000, (b,e) DIC, (c,f) CFAIC, (d,g) PCFAIC.

Fig.7 depicts enlarged parts of the images cropped in areas with significant structural content. The results correspond to the compression ratio 30 . Visual inspection of the results reveals that the JPEG-based solutions produce the final images which suffer from blocking effects and color shifts, whereas solutions equipped with JPEG 2000 produce images with enhanced sharpness. It should be also noted that employing the $\mathrm{CCA}$ demosaicking solution results in true coloration and preserved structural content. On the other hand, the use of the BI demosaicking solution is associate with edge blurring, aliasing effects and color artifacts. The interesting reader can find additional material and results in the full-length paper of [7].

\section{Conclusions}

Single-sensor image processing pipelines based on the popular Bayer CFA were presented. Considered solutions can store either the CFA data or the demosaicked fullcolor data, thus resulting in the different visual quality and computational efficiency. Evaluating the final images from the end-user's perspective indicates that JPEG 2000-based CFA image compression solutions can be an essential element in the next generation of single-sensor digital cameras.

\section{References}

[1] Technical Standardization Committee on AV \& IT Storage Systems and Equipment, "Exchangeable image file format for digital still cameras: Exif Version 2.2," JEITA CP-3451, April 2002.

[2] Technical Committee ISO/TC 42, Photography, "Electronic still picture imaging - Removable memory, Part 2: Image data format - TIFF/EP," ISO 12234-2, January 2001.

[3] R. Lukac and K. N. Plataniotis, "Color filter arrays: Design and performance analysis," IEEE Trans. Consumer Electronics, vol. 51, pp. 1260-1267, Nov. 2005.

[4] R. Lukac, K. N. Plataniotis, and D. Hatzinakos, "Color image zooming on the Bayer pattern," IEEE Trans. Circuit and Systems for Video Technology, vol. 15, pp. 1475-1492, Nov. 2005.
[5] K. Parulski and K. E. Spaulding, "Color image processing for digital cameras," in Digital Color Imaging Handbook, (eds.) G. Sharma, CRC Press, Boca Raton, FL., pp. 728-757, 2002.

[6] R. Lukac and K. N. Plataniotis, "Data-adaptive filters for demosaicking: A framework," IEEE Trans. Consumer Electronics, vol. 51, pp. 560-570, May 2005.

[7] R. Lukac and K. N. Plataniotis, "Single-sensor camera image compression," IEEE Trans. Consumer Electronics, submitted.

[8] Y. T. Tsai, "Color image compression for single-chip cameras," IEEE Transactions on Electron Devices, vol. 38, no. 5, pp. 1226-1232, May 1991.

[9] S. Battiato, A. R. Bruna, A. Buemi, and A. Castorina, "Analysis and characterization of JPEG 2000 standard for imaging devices," IEEE Trans. Consumer Electronics, vol. 49, pp. 773-779, Nov. 2003.

[10] T. Toi and M. Ohta, "A subband coding technique for image compression in single CCD cameras with Bayer color filter arrays," IEEE Trans. Consumer Electronics, vol. 45, pp. 176-180, Feb. 1999.

[11] C. C. Koh, J. Mukherjee, and S. K. Mitra, "New efficient methods of image compression in digital cameras with color filter array," IEEE Trans. Consumer Electronics, vol. 49, pp. 1448-1456, Nov. 2003.

[12] N. Zhang and X. Wu, "Lossless compression of color mosaic images," in Proc. IEEE International Conference on Image Processing (ICIP'04), vol. 1, pp. 517520, October 2004.

[13] A. Bazhyna, A. Gotchev, K. Egiazarian, "Near-lossless compression algorithm for Bayer pattern color filter arrays," SPIE-ISET Electronic Imaging: Digital Photography, vol. 5678, pp. 198-209, 2005.

[14] T. Sakamoto, C. Nakanishi, and T. Hase, "Software pixel interpolation for digital still cameras suitable for a 32-bit MCU," IEEE Trans. Consumer Electronics, vol. 44, pp. 1342-1352, Nov. 1998.

[15] R. Lukac, K. N. Plataniotis, D. Hatzinakos, and M. Aleksic, "A novel cost effective demosaicing approach," IEEE Trans. Consumer Electronics, vol. 50, pp. 256261, Feb. 2004. 